\title{
LXIV. On the retardation of alpha rays by metals and gases
}

\section{T.S. Taylor}

To cite this article: T.S. Taylor (1909) LXIV. On the retardation of alpha rays by metals and gases, Philosophical Magazine Series 6, 18:106, 604-619, DOI: $10.1080 / 14786441008636732$

To link to this article: http://dx.doi.org/10.1080/14786441008636732

曲 Published online: 21 Apr 2009.

Submit your article to this journal $\pi$

Џ Article views: 3

Q View related articles $\square$

Citing articles: 8 View citing articles 5 


\section{[ 604 ] \\ LXIV. On the Retardation of Alpha Rays by Metals and Gases. By T. S. TAYLOR* \\ Introduction.}

TN a preliminary paper + " On the Retardation of Alpha 1 Rays by Metal Foils and its Variation with the Speed of the Alpha Particles," the writer described some experiments which showed clearly that the air-equivalents of metal foils decrease with the range of the alpha-particles entering the foils $\ddagger$. By "air-equivalent" is meant the amount by which the range of the alpha-particles in air is cut down by their passage through the foil. It was shown that the change in the air-equivalents is small for thin foils of the lighter metals when the speed of the alpha-particles entering the sheets is high : but, when the speed of the particles is low for thin sheets or, when the sheets are thicker, the change becomes quite marked. A comparison of the change for sheets of different metals of nearly equal air-equivalents showed the rate of change to be in the order of the atomic weights of the metals. The results obtained in these experiments were not sufficient to furnish an explanation of the phenomenon; but the continuation of the experiments during the last year under somewhat different conditions has furnished results which do lead to conclusions of some interest.

\section{Scattering of the Alpha Rays.}

In the determination of the variation in the air-equivalents with the speed of the alpha-particle, as described in the paper cited above, the source of rays (polonium) with the metal sheet over it was set at such a distance from the ionization-chamber that some part of the top, or nearly horizontal, portion of the Bragg ionization-curve fell within the ionization-chamber. A slight increase in the range of the particle in this portion of curve corresponds to a considerable increase in the ionization. With the polonium set

* Communicated by Professor H. A. Bumstead.

+ American Journal of Science, vol. xxvi. pp. 169-179, Sept. 1908.

I The phenomenon upon which this work was based was first observed by Mme Curie and has later been investigated by several others. Bragg \& Kleeman (Phil. Mag. Sept. 1005, and April 1907) observed that the stopping power of a metal was not independent of the speed. Kucera \& Masek (Phys. Zeitschrift, xix. pp. 630-40, 1906) and Meitner (Phys. Zeitschrift, viii. p. 489,1907 ) ascribe the effect to a difference in the amount of scattering. McClung (Phil. Mag. Jan. 1906), Rutherford (Phil. Mag. Aug. 1906), and Levin (Phys. Zeitschrift, xv. pp. 519-521, 1906) obtained results which indicate that each successive layer of aluminium foil diminishes the range of the $a$-particle by the same amount. 
at a definite distance from the ionization-chamber, it was found that, when the metal sheet was moved away from the polonium towards the ionization-chamber, the ionization increased. This increase in the ionization was attributed to the alpha-particle having a greater velocity (or range) upon entering the chamber when the sheet was near the chamber than it had when the sheet was at a distance from the chamber. Hence the metal sheet did not cut down the range of the particle so much when the sheet was at a distance from the polonium as it did when near the polonium. As a preliminary to more extensive experiments by this method two tests were made to ascertain whether a scattering of the rays could explain the increase in the ionization observed when the metal sheets were moved away from the polonium towards the ionization-chamber.

First test.-Any marked scattering of the rays by the foils would change the shape of the cone of rays, and especially the form of the top portion of the cone. The slope of the top, or nearly horizontal portion, of the Bragg ionizationcurve, as well as the value of the maximum ionization, depends upon the form of the cone of rays arriving at the ionizationchamber. Thus, if scattering of the rays exists to a very marked degree, it might be expected that differences between the slope and form of the two Bragg curves obtained with and without the metal foil over the polonium could be readily detected. With polonium as the source of rays, numerous determinations of the Bragg curves, both with and without the various foils over the polonium, were made. A study of these curves showed them to run parallel to each other and to give the same value of the maximum ionization. The effect of putting the foils over the polonium was merely to diminish all the ordinates of the curves by the same amount.

Second test.-An iris diaphragm whose circular opening could be adjusted to any desired diameter between $\frac{1}{2}$ and $5 \frac{1}{2} \mathrm{crn}$., was constructed of thin sheets of brass and placed directly below the ionization-chamber. The centre of the opening of the diaphragm was directly below the centre of the ionization-chamber. With the source of rays (radium $\mathrm{C}$ ) at such a distance from the ionization-chamber that the chamber cut the top portion of the Bragg curve, the ionization was measured for various distances of the metal sheets above the source of rays; first with the diaphragm entirelv open and then with the opening in the diaphragm of such diameter as to just limit the geometrical beam of rays, or to cut off the edge of the beam. For any given position of the sheet above the source of rays, the ionization was always 
greater when the diaphragm was completely open than it was when the diaphragm just limited the beam. However, the difference between the ionization in the two cases was a constant value for all positions of the metal sheets above the source of rays. This difference would not be a constant quantity if the scattering of the rays was the occasion of the increase in the ionization produced by moving the metal sheets away from the source of the rays. On the contrary, the difference between the ionizations with and without the diaphragm limiting the geometrical beam of rays would be greater when the sheet is far away from the source of rays than when it is pear the source of rays if scattering of the rays by the foils was the cause of the increase in the ionization. The fact that the ionization was greater with the diaphragm open than when it just limited the cone of rays signifies that more alpha-particles get into the ionizationchamber in the former than in the latter case, and therefore confirms the existence of the scattering of the rays by metal foils as found by Geiger *.

These two methods of investigation, altbough in the case of the latter showing the existence of the scattering of the rays, seem to be sufficient to preclude scattering as an explanation for the so-called decrease in the air-equivalents of the metal sheets as they are moved away from the polonium. By measuring the ionizations with and without the diaphragms limiting the cone of rays when there was not a metal sheet over the source of rays, it was found that the ionization was greater in the latter than in the former case, which shows that the rays are scattered by air as well as by metals. These methods, however, are not particularly suitable for measuring the amount of the scattering, and hence no comparison as to how much each metal scatters the rays was attempted. The important fact is that the effect under consideration is not influenced by the scattering of the rays.

\section{Continuation of the Experiments.}

In the first experiments polonium had been used as the source of rays, but in order to extend the study to alphaparticles of higher range, radium $\mathrm{C}$ has been used in the present experiments. This made it possible to use foils of greater thickness than had been previously used. A thin aluminium foil covered with a thin coating of lacquer was put directly over a capsule containing a thin film of pure radium bromide in order to prevent escape of the emanation. The bole in the brass plug over the radium bromide was of

* Proceedings of the Royal Society, Series A, vol. lxxxi. no. A 546 p. 174 . 
such dimensions that the cone of rays emerging from it fell well within the limits of the ionization-chamber. The radium bromide was set at such a distance from the ionizationchamber that a part of the top, or slightly inclined portion, of the Bragg ionization-curve due to the alpba-particles from radium $\mathrm{C}$ fell within the chamber, and the air-equivalents of the varions metal sheets determined at various points in the path of the rays in exactly the same manner as that used in the first experiments *. The rays of shorter range than those of radium $\mathrm{C}$ had no effect upon the results since they did not reach the ionization-chamber. Beta and gamma rays are also given off by the radium bromide, but the ionization produced by them can be considered as a constant value over the part of the path used, and consequently the effect due to them is only a shifting of the curves to the right parallel to themselves which would have noeffect upon the results under consideration.

In column 1, Table I., are given the different metal sheets used in the experiments. Column 2 contains the thickness in

\section{TABLE I.}

\begin{tabular}{|c|c|c|c|}
\hline I. & II. & $\begin{array}{c}\text { III. } \\
\text { Mir-Equivalent } \\
\text { in cros. }\end{array}$ & IV. \\
Ratio.
\end{tabular}

* Loc. cit. pp. 173-175. 
centimetres of the respective sheets. Column 3 has the airequivalents of the sheets as measured directly when the sheets were nearest the source of rays. Column 4 contains the ratios of the air-equivalents to the thickness of the respective metals. The last three lines of the table will be referred to later. The air-equivalents of the sheets given in column 1 , Table I., as determined by an improved method for any position of the sheets are given in 'lable II.

TABLE IL.

\begin{tabular}{|c|c|c|c|c|c|c|c|c|}
\hline $\begin{array}{c}\text { Range in cms. } \\
\text { of entering } \\
a \text {-particle. }\end{array}$ & A Al. & B Al. & C Al. & D Al. & A A Au. & $\mathrm{BAu}$. & O Au. & DAu. \\
\hline $\begin{array}{l}5 \cdot 7 \\
5 \cdot 3 \\
4 \cdot 9 \\
4 \cdot 5 \\
4 \cdot 1 \\
3 \cdot 7 \\
3 \cdot 3 \\
2 \cdot 9 \\
2 \cdot 5 \\
2 \cdot 1 \\
1 \cdot 7 \\
1 \cdot 3\end{array}$ & $\begin{array}{l}0.597 \\
0.597 \\
0.597 \\
0.597 \\
0.597 \\
0.597 \\
0.596 \\
0.595 \\
0.592 \\
0.584 \\
0.570 \\
0.555\end{array}$ & $\begin{array}{c}1 \cdot 209 \\
1 \cdot 206 \\
1 \cdot 202 \\
1 \cdot 198 \\
1 \cdot 193 \\
1 \cdot 187 \\
1 \cdot 178 \\
1 \cdot 168 \\
1 \cdot 152 \\
1 \cdot 131 \\
\ldots\end{array}$ & $\begin{array}{c}1 \cdot 803 \\
1 \cdot 792 \\
1.781 \\
1.769 \\
1.757 \\
1.742 \\
1.724 \\
1.705 \\
\ldots \\
\ldots \\
\ldots\end{array}$ & $\begin{array}{l}2 \cdot 672 \\
2 \cdot 659 \\
2 \cdot 644 \\
2 \cdot 628 \\
2 \cdot 608 \\
2.582 \\
\ldots \\
\ldots \\
\ldots \\
\ldots \\
\ldots\end{array}$ & $\begin{array}{l}0 \cdot 719 \\
0 \cdot 706 \\
0.603 \\
0 \cdot 6<0 \\
0 \cdot 668 \\
0.657 \\
0.644 \\
0.630 \\
0 \cdot 616 \\
0 \cdot 600 \\
0.582\end{array}$ & $\begin{array}{l}0.980 \\
0.966 \\
0.947 \\
0.929 \\
0.910 \\
0.889 \\
0.866 \\
0.837 \\
0.805 \\
0.757\end{array}$ & $\begin{array}{l}1.375 \\
1.352 \\
1.328 \\
1.303 \\
1.276 \\
1.248 \\
1.214 \\
1.178 \\
1.127\end{array}$ & $\begin{array}{l}1.960 \\
1.869 \\
1.837 \\
1.802 \\
1.763 \\
1.720 \\
1.659 \\
1.598\end{array}$ \\
\hline $\begin{array}{l}\text { Range in cms. } \\
\text { of entering } \\
a-\text { particle. }\end{array}$ & A Sn. & B Sn. & $\mathrm{A} \mathrm{Pb}$. & $\mathrm{B} \mathrm{Pb}$. & $\mathrm{C} \mathrm{Pb}$. & Paper. & Cell & lloidin. \\
\hline $\begin{array}{l}5 \cdot 7 \\
5 \cdot 3 \\
4 \cdot 9 \\
4 \cdot 5 \\
4 \cdot 1 \\
3 \cdot 7 \\
3 \cdot 3 \\
2 \cdot 9 \\
2 \cdot 5 \\
2 \cdot 1 \\
1 \cdot 7 \\
1 \cdot 3\end{array}$ & $\begin{array}{l}1 \cdot 011 \\
1 \cdot 002 \\
0.993 \\
0.984 \\
0.971 \\
0.957 \\
0.941 \\
0.923 \\
0.902 \\
0.882 \\
0.853\end{array}$ & \begin{tabular}{c|c}
1 & 1.995 \\
2 & 1.980 \\
3 & 1.960 \\
4 & 1.988 \\
1 & 1.914 \\
7 & 1.884 \\
1 & 1.845 \\
3 & $1 \cdot 795$ \\
2 & $\ldots$ \\
2 & $\ldots$ \\
3 &
\end{tabular} & $\begin{array}{l}1 \cdot 104 \\
1.085 \\
1.064 \\
1.042 \\
1.020 \\
0.999 \\
0.977 \\
0.950 \\
0.923 \\
0.888\end{array}$ & \begin{tabular}{l|l}
4 & 1.396 \\
5 & 1.371 \\
4 & 1.343 \\
2 & 1.320 \\
0 & 1.289 \\
9 & 1.263 \\
7 & $1 \cdot 232$ \\
9 & 1.200 \\
3 & $1 \cdot 163$ \\
8 &
\end{tabular} & $\begin{array}{l}2 \cdot 325 \\
2 \cdot 296 \\
2 \cdot 260 \\
2 \cdot 220 \\
2 \cdot 180 \\
2 \cdot 124 \\
2 \cdot 046\end{array}$ & $\begin{array}{c}1 \cdot 020 \\
, " \\
", \\
", \\
", \\
", \\
"\end{array}$ & & $\begin{array}{c}0 \cdot 520 \\
: \\
" \\
" \\
" \\
" \\
" \\
" \\
" \\
"\end{array}$ \\
\hline
\end{tabular}

The aluminium foil over the radium to prevent the escape of the emanation cut down the range of the alpha-particles $0.46 \mathrm{~cm}$. The height of the plug containing the radium was $0.9 \mathrm{~cm}$. above the radium. Therefore the maximum available range of the alpha-particles entering the sheet is $5 \cdot 70$. The values of the air-equivalents for each of the metal sheets in Table II. represent the average results obtained from a series of from six to ten separate determinations, the details of which have been omitted for the sake of brevity. 
Experiments were also made with sheets of paper and celloidin*. Two sheets of paper of about one and two $\mathrm{cm}$. air-equivalents respectively, and three sheets of celloidin of air-equivalents of the order of $0.5,1.0$ and $2.0 \mathrm{~cm}$. respectively were used. For these sheets of paper and celloidin, the ionization did not increase as the sheets were moved away from the radium but had the same value for all positions of the sheets and hence their air-equivalents remained constant.

The behaviour of the sheets of paper and celloidin, the atomic weights $\dagger$ of which are about the same as that of air, suggested the idea of undertaking to obtain sheets of some substance such as hydrogen, whose atomic weight is less than that of air. For this purpose a ring about one centimetre wide was cut from a brass tube six centimetres in diameter and two small brass tubes were put in the ring diametrically opposite each other. Thin films of celloidin were stretched across each side of the ring and held in place by universal wax. This formed a cell which could be filled with hydrogen and then used in the same manner as the metal foils. To be certain that the cell was always full of hydrogen a slight current of the gas was kept flowing through it all the time during an experiment. A current of air was kept circulating through the case surrounding the apparatus in order to prevent the hydrogen that might possibly leak from the cell from entering the ionization-chamber. ' The air-equivalent of the hydrogen cell, or sheet, when $0.9 \mathrm{~cm}$. from the radium was determined by plotting the ionization-curve first with hydrogen and then with air in the cell. The ordinates of the latter curve were all increased by the thickness of the cell of hydrogen which gave the position of the curve if the cell had been evacuated. The difference between the ordinates of the two curves corresponding to a given abscissa was the airequivalent of the hydrogen sheet.

When the cell containing the hydrogen was moved away from the radium, which was kept at a given position as in the previous cases, it was found that the ionization decreased, which signified that the total range in air of the alpha-particle was less when the hydrogen sheet was far away from the radium than when it was near the radium. Thus the amount by which the range of the alpha-particle was cut down by its passage through the cell was greater when the cell was at a distance from the radium than it was when it was near the radium. Consequently the air-equivalent of the

* Celloidin is a specially pure preparation of collodion.

$\dagger$ By atomic weight of air, paper, and celloidin is meant the average weight of the constituent atoms. 
hydrogen cell increased as the range of the entering alphaparticle decreased. The particles had to pass through the celloidin sheets but this did not influence the effect because, as we have seen, the amount by which the range was cut down by the celloidin sheets was constant for all positions of the celi. Determinations of the air-equivalents in centimetres of three hydrogen cells given in 'Table I. were made for various distances of the cell from the radium and the results obtained are recorded in Table III.

TABLE III.

\begin{tabular}{|c|c|c|c|}
\hline $\begin{array}{c}\text { Range of the } \\
\text { a-particle upon } \\
\text { entering the } \\
\text { bydrogen. }\end{array}$ & A Hydrogen. & B Hydrogen. & C Hydrogen. \\
\hline $5 \cdot 2$ & $0 \cdot 231$ & $0 \cdot 428$ & $0 \cdot 762$ \\
$4 \cdot 8$ & $0 \cdot 235$ & $0 \cdot 434$ & $0 \cdot 776$ \\
$4 \cdot 4$ & $0 \cdot 241$ & $0 \cdot 442$ & $0 \cdot 791$ \\
$4 \cdot 0$ & $0 \cdot 247$ & 0.451 & 0.807 \\
$3 \cdot 6$ & $0 \cdot 254$ & $0 \cdot 460$ & $0 \cdot 831$ \\
$3 \cdot 2$ & $0 \cdot 262$ & $0 \cdot 470$ & 0.861 \\
$2 \cdot 8$ & $0 \cdot 271$ & $0 \cdot 483$ & 0.896 \\
$2 \cdot 4$ & $0 \cdot 283$ & 0.499 & 0.938 \\
\hline
\end{tabular}

The reason the maximum range here is $5.2 \mathrm{~cm}$. instead of $5.7 \mathrm{~cm}$. as it was in Table II. is because the air-equivalent of the lower film of celloidin must be subtracted since the alpha-particles must pass through it before entering the hydrogen. The air-equivalent of the lower film was $0.5 \mathrm{~cm}$.

Althongh the air-equivalents of the celloidin sheets remained constant, it seemed probable, from the behaviour of the hydrogen sheets, that if the same experiments were performed in an atmosphere of hydrogen, the hydrogenequivalent * of the celloidin sheet would not remain constant, but would decrease as the range of the alpha-particles decreased. To investigate this point the apparatus was enclosed in an air-tight sheet-iron case, which by several partial evacuations and refillings could be filled with practically pure hydrogen. With polonium as the source of rays the hydrogen equivalents in $\mathrm{cms}$. of sheets of celloidin, aluminium, tin, and gold were determined for various distances of the sheets from the polonium. Only the results for the celloidin and A gold are given in Table IV. as they are sufficient to illustrate the point in question.

* The hydrogen-equivalent is the amount by which the range of the $a$-particles in hydrogen is cut down by their passage through the sheet. 
Alpha Rays by Metals and Gases.

611

Table IV.

\begin{tabular}{|c|c|c|c|c|c|c|c|c|c|c|}
\hline $\left.\begin{array}{c}\text { Range in } H \\
\text { of entering } \\
\text { particle ... }\end{array}\right\}$ & $13 \cdot 0$ & $12 \cdot 6$ & $12 \cdot 2$ & $11 \cdot 8$ & $11 \cdot 4$ & $11 \cdot 0$ & $10 \cdot 6$ & $10 \cdot 2$ & $9 \cdot 8$ & $9 \cdot 4$ \\
\hline Celloidira ...... & $23 \cdot 20$ & $23 \cdot 08$ & $22 \cdot 96$ & $22 \cdot 80$ & 2264 & $22 \cdot 48$ & $22 \cdot 32$ & $22 \cdot 16$ & 21.88 & $21 \cdot 60$ \\
\hline A Gold.. & $2 \% \cdot 97$ & $27 \cdot 55$ & $27 \cdot 11$ & $26 \cdot 67$ & $26 \cdot 23$ & $25 \cdot 75$ & $25 \cdot 17$ & $24 \cdot 49$ & 2391 & $23 \cdot 01$ \\
\hline
\end{tabular}
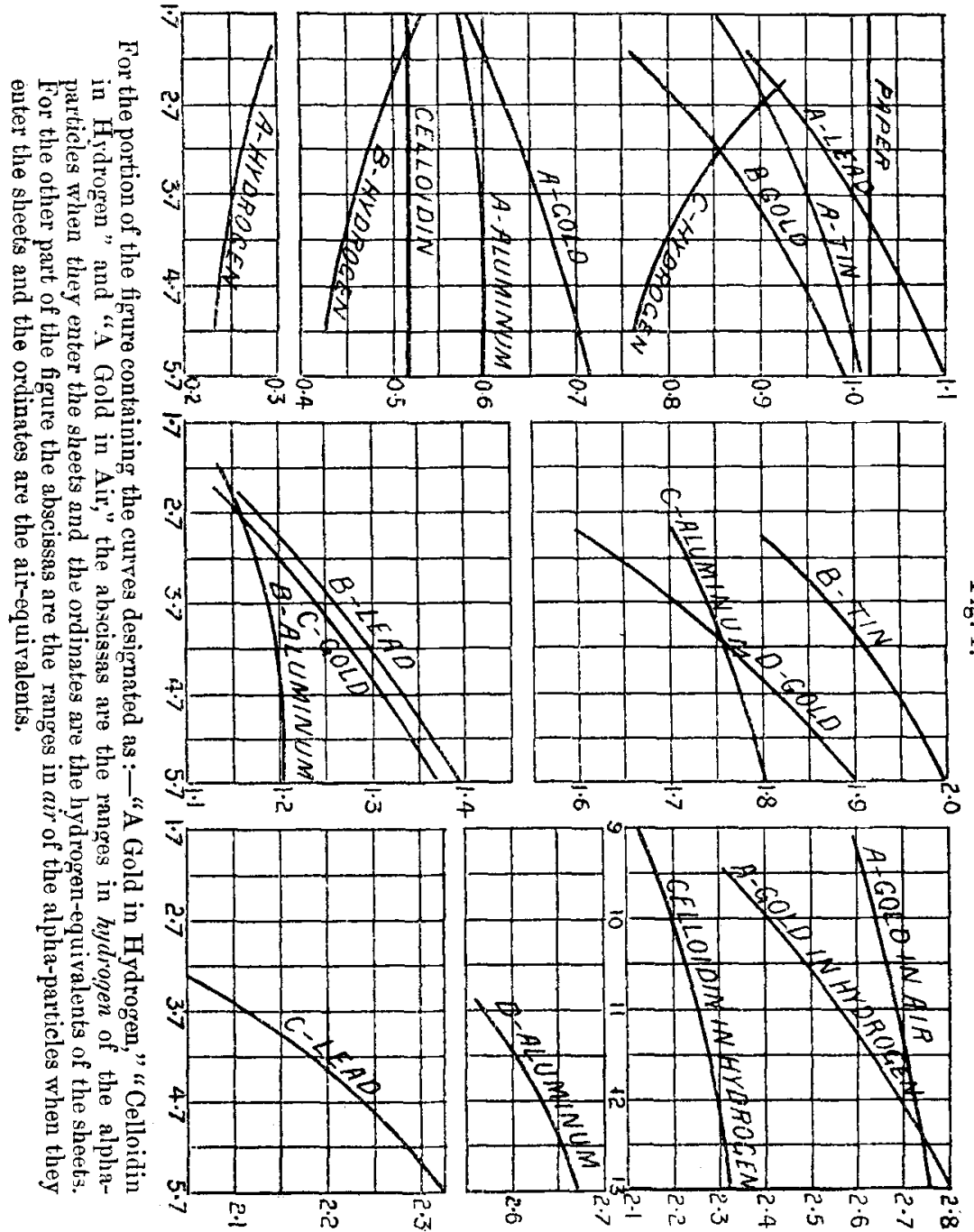
The curves in figure 1 (p. 611) represent the results recorded in Tables II., III., and IV. By noting the slopes of the curves some comparison of the rates at which the airequivalents of the various sheets change can be obtained.

Taking the general slope of each curve and dividing it into the air-equivalent of the corresponding sheet when $0.9 \mathrm{~cm}$. from the radium, it is found that for a given metal the quotient thus obtained is nearly constant for all the sheets of the metal. This is shown in column 4, Table V. Thus for

TABLE $V$.

\begin{tabular}{|c|c|c|c|c|c|}
\hline Sheets. & $\begin{array}{l}\text { Slopes of } \\
\text { Curves. }\end{array}$ & $\begin{array}{c}\text { Air- } \\
\text { equivalents. }\end{array}$ & Ratio. & $\sqrt{\text { atomic weight. }}$ & ${ }_{\text {ratio }}^{\text {Mean }} \sqrt{\text { atomic wt. }}$ \\
\hline $\begin{array}{lll}\text { A } & A u & \ldots \\
\text { B } & A u & \ldots \\
\text { C } & A u & \ldots \\
\text { D } & A u & \ldots \\
\text { A } & \text { Sn } & \ldots \\
\text { B } & \text { Sn } & \ldots \\
\text { A } & \text { Pb } & \ldots \\
\text { B } & \text { Pb } & \ldots \\
\text { C } & \text { Pb } & \ldots \\
\text { A } & A 1 & \ldots \\
\text { B } & A 1 & \ldots \\
\text { C } & A 1 & \ldots \\
\text { D } & \text { Al } & \ldots \\
\text { A } & \text { H } & \ldots \\
\text { B } & \text { H } & \ldots \\
\text { C } & \text { H } & \ldots\end{array}$ & $\begin{array}{r}0.032 \\
0.051 \\
0.064 \\
0.100 \\
0.032 \\
0.063 \\
0.053 \\
0.071 \\
0.110 \\
0.010 \\
0020 \\
0.033 \\
0.045 \\
-0.020 \\
-0.034 \\
-0.064\end{array}$ & $\begin{array}{l}0 \cdot 719 \\
0.980 \\
1.375 \\
1.900 \\
1 \cdot 011 \\
1 \cdot 995 \\
1 \cdot 104 \\
1 \cdot 396 \\
2 \cdot 325 \\
0 \cdot 597 \\
1 \cdot 209 \\
1 \cdot 803 \\
2 \cdot 67.2 \\
0 \cdot 231 \\
0 \cdot 428 \\
0 \cdot 762\end{array}$ & $\begin{array}{l}2 \cdot 24 \times 10^{1} \\
1.92 \times 10^{1} \\
\cdot 15 \times 10^{1} \\
1.90 \times 10^{1} \\
3 \cdot 16 \times 10^{1} \\
3.17 \times 10^{1} \\
2 \cdot 08 \times 10^{1} \\
1.96 \times 10^{1} \\
2 \cdot 11 \times 10^{1} \\
5 \cdot 97 \times 10^{1} \\
6 \cdot 04 \times 10^{1} \\
5 \cdot 48 \times 10^{1} \\
5 \cdot 93 \times 10^{1} \\
1 \cdot 16 \times 10^{1} \\
1 \cdot 17 \times 10^{1} \\
1 \cdot 19 \times 10^{1}\end{array}$ & $\begin{array}{l}14.05 \\
10.91\end{array}$ & $\begin{array}{l}28 \cdot 80 \\
34 \cdot 34\end{array}$ \\
\hline
\end{tabular}

sheets of the same metal the rate at which the arr-equivalent of each sheet changes with a change in the range of the entering alpha-particles, is proportional to its air-equivalent when nearest the radium. The approximately constant numbers in column $\hat{b}$, Table $V$., show that the percentage rate of change in the air-equivalent for any metal is nearly proportional to the square root of the atomic weight. The agreement of the values in columns 4 and 6 , Table V., is as good as could be expected since the slopes of the curves in figure 1 could only be determined roughly. The proportionality is indeed only approximate, since the curve for any one sheet does not have a constant slope.

For the thin sheet of aluminium the air-equivalent is almost constant for the higher ranges or speeds, but as the speed of the entering alpha-particle decreases the air-equivalent decreases slowly, and in the lower ranges the decrease becomes quite apparent. For the thicker sheets of aluminium the change is more marked even for the higher ranges. The statements of Mcolung, Levin, and Rutherford that equal 
successive layers of aluminium foil diminish the range of the alpha-particles by equal amounts seem to hold true for thin sheets of foil when the range is high; but when the metal sheet is thicker, or for thin sheets when the range is low, it does not hold. The slight difference, however, in the airequivalent of the thin foil, when near and far away from the polonium, would scarcely be detected by measuring directly the air-equivalent in the two positions. This is probably the explanation of the above statements by McClung, Levin, and Rutherford.

Since the air-equivalent of a metal sheet decreases with the speed of the alpha-particle entering it, the ratio of the air-equivalent to the thickness of a given sheet of metal should be less than the same ratio for a thinner sheet of the same metal. This is shown to be true by the last column of Table I. For the hydrogen sheets on the contrary the same ratio should increase as the thickness of the cell or sheet of hydrogen increases. This is also confirmed by the last column of Table I.

While the air-equivalent of the sheet of celloidin remains constant the hydrogen-equivalent of the same does not remain constant but decreases as the range of the alpha-particle in hydrogen decreases. The curve "Celloidin in Hydrogen," fig. 1, which was plotted from the results recorded in Table IV., illustrates this point. It is to be noted also from the curve "A Gold in Hydrogen" (fig. 1) that the rate at which the hydrogen-equivalent of the A gold decreases is much greater than the rate at which its air-equivalent decreases. The curve designated "A Gold in Air" (fig. 1) is the portion of the "A Gold" curve in the same figure that lies to the left of the abscissa 3.0 . The co-ordinates of that portion of the curve are magnified about $4 \frac{2}{3}$ times so as to be plotted on the same scale as the curves obtained in the hydrogen atmosphere. $4 \frac{2}{3}$ is the ratio of the thickness of a hydrogen sheet to its air-equivalent when near the radium. The slope of the curve "A Gold in Air" is practically the same as that of "Celloidin in Hydrogen" as can be seen from the figure. The angle which the curve "A Gold in Hydrogen" makes with the curve "A Gold in Air" is about the same as the angle which the curve "Celloidin in Hydrogen" makes with the axis of abscissas. The slope of the curve "A Gold in Hydrogen" is nearly $3 \frac{3}{4}$ times the slope of the curve "Celloidin in Hydrogen." But $3 \frac{3}{4}$ is the ratio of the square root of the atomic weight of gold to that of air

$$
\left[\sqrt{\frac{197}{14}}=3 \cdot 75+\right] \text {. }
$$


Hence the rates, at which the hydrogen-equivalents of the Gold and Celloidin sheets decrease with the speed of the alpha-particle entering the sheets, are proportional to the square roots of their respective atomic weights. Moreover the slope of the curve "Celloidin in Hydrogen" is numerically equal (but of opposite sign) to the slope of the curve "B Hydrogen" in air. The hydrogen-equivalent of the celloidin sheet was somewhat larger than the thickness of the "B Hydrogen" cell, but it seems entirely proper to conclude that the rate, at which the hydrogen-equivalent of the celloidin sheet decreases with the speed of the alpha-particle, is the same as the rate at which the air-equivalent of the "B Hydrogen" increases as the speed of the entering alphaparticle decreases.

The possibility that the observed variations in the ionization, which have been taken to be the measures of the changes in the air-equivalents, may be due to secondary rays is precluded by the fact that numerous direct determinations of the Bragg ionjzation-curves, with and without the metal sheets near the polonium, and again near the ionization-chamber, showed no irregularities in the curves as would be expected were secondary rays present in any appreciable amount. The behaviour of the air-equivalents of the hydrogen sheets in no way conforms to what might be expected to be produced by secondary rays.

The increasing of the air-equivalents of the hydrogen sheets and the decreasing of the hydrogen-equivalents of the celloidin sheets when moved away from the source of rays gave occasion for suspecting that $s$ me differences might be found to exist between the Bragg ionization-curves obtained in atmospheres of air and hydrogen respectively. To determine these curves, use was made of an apparatus constructed for Mr. F. E. Wheelock, of this Laboratory, which was similar to the one used thus far in the work, except that the vessel enclosing the main part of the apparatus could be completely exhausted. To make any comparison of the two ionization curves, it was necessary to determine them under similar conditions-i.e., the same source of rays was used in the two cases, and the pressure of the air was so reduced as to make the range of the alpha-particles in air equal to their range in hydrogen at normal pressure. Polonium was used as the source of rays, and several Bragg curves were obtained in hydrogen at normal pressure and in air at a reduced pressure of about $17 \mathrm{~cm}$. of mercury. Two of the curves are shown in fig. 2. The dotted portion of each curve is assumed to be of 
the form it would take were it possible to move the polonium entirely up to the ionization-chamber. At all events these assumed portions of the curves can differ but little from what the actual curves would be.

Fig. 2.

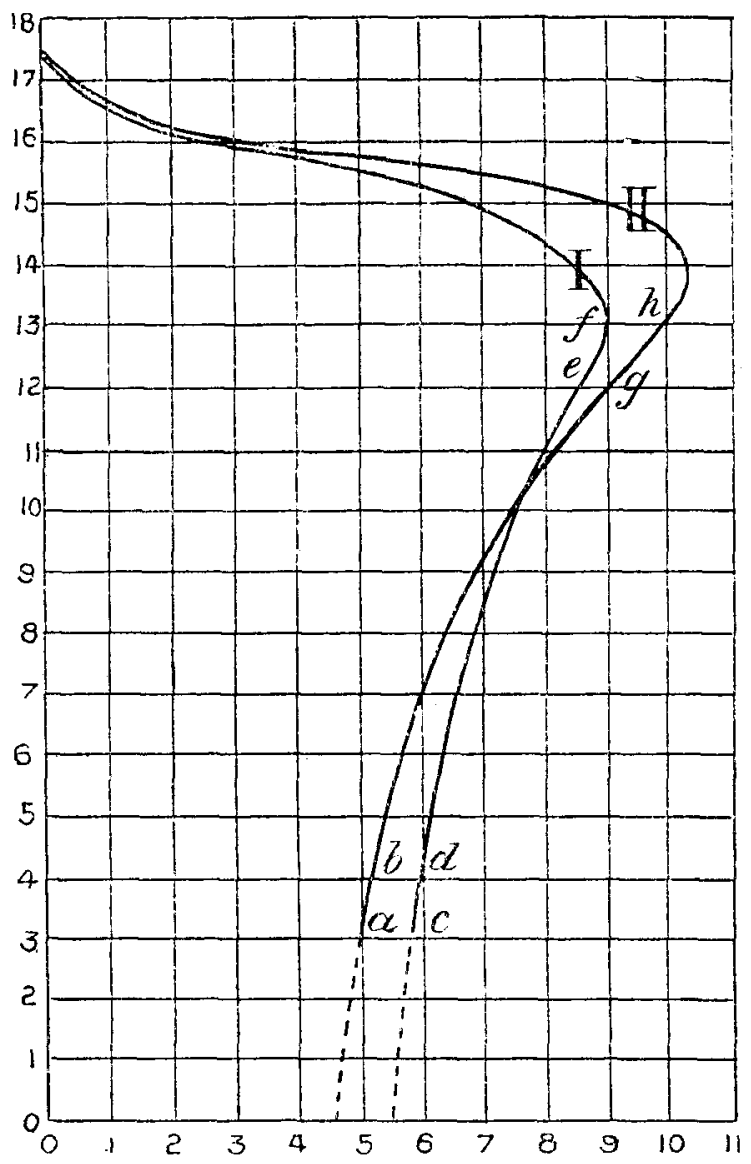

The ordinates of the curves are the distances in centimetres of the polonium from the ionization-chamber. The abscissas are the deflexions in centimetres of the electrometer-needle per second. Curve I. was obtained in air at a reduced pressure of about 17 centimetres of mercury. Curve II. was obtained in hydrogen at normal pressure.

It is to be observed that the two eurves in fig. 2 present slight differences in form. The probable interpretation of these differences will now be considered. Any given abscissa 
of either curve is a measure of the ionization produced by the particles in the gas in the chamber when the polonium was at a distance from the chamber represented by the ordinate corresponding to the given abscissa. Consequently, the total area enclosed by the two axes of reference and either curve is proportional to the total ionization produced in the gas in wbich the curve was determined. By measuring these areas with a planimeter, it was found they were equal. This confirms the observations by Bragg * that the total ionization produced by the alpha-particle in air is the same as that in hydrogen. From the curves of fig. 2 it is seen that when the speed of the alpha-particle is high more ions are produced per centimetre of path in air than in hydrogen, but when the speed is low more ions are produced per centimetre in hydrogen than in air.

Let us suppose that for a given speed of the alpha-particle the amount of energy required to produce an ion is the same in all substances. Then for air we would have the relation

$$
d \mathrm{I}_{a}=-f(\mathrm{~V}) d \mathrm{E}_{a} .
$$

The corresponding relation in hydrogen is

$$
d \mathrm{I}_{h}=-f(\mathrm{~V}) d \mathrm{E}_{h} .
$$

Dividing the former by the latter, we have

$$
\frac{d \mathrm{I}_{a}}{d \mathrm{I}_{h}}=\frac{f(\mathrm{~V}) d \mathrm{E}_{a}}{f(\mathrm{~V}) d \mathrm{E}_{h}}
$$

which for a given speed $\mathrm{V}$ in each gas reduces to

$$
\frac{d \mathrm{I}_{a}}{d \mathrm{l}_{h}}=\frac{d \mathrm{E}_{a}}{d \mathrm{E}_{h}}
$$

From this it is seen that for a given speed of the alphaparticle the ratio of the rates of the consumption of the energy in producing ions in air and bydrogen, is equal to the ratio of the rates at which the ionization is produced in the respective gases. On the basis of our hypothesis, let us consider the ratios of the energies consumed in the 4th and 13th centimetres (fig. 2) of the path of the particle in air and hydrogen. This ratio for the 4 th centimetre of the path is proportional to $\frac{\text { area } c d 43 c}{\text { area } a b 43 a}$, since the areas are proportional to the ionizations produced in the gases. The corresponding ratio for the 13th centimetre is equal to $\frac{\text { area } e, f, 13,12, e}{\text { area } g, h, 13,12, g}$. The former ratio is seen from the figure to be greater than the * Phil. Mag. March 1907, p. 333. 
latter. Moreover it is also seen that the ratio of the energy of the alpha-particle absorbed by any given centimetre of air to the energy absorbed by the corresponding centimetre of hydrogen, is always greater than the corresponding ratio for the centimetre just beyond the given one. This is in agreement with the results obtained for the air-equivalents of the hydrogen cells ; because the increase in their air-equivalents as the range decreases is due to the fact that the ratio of the energy absorbed by the hydrogen cell to the energy that would be consumed by the air which it displaces, continually increases as the cell is moved away from the source of rays. The thicker the cell the more rapid would be the rate of increase, as could be seen by comparing the areas which represent the ionizations in, say, 2 centimetres of air and hydrogen respectively in fig. 2 in two different positions. The increase in the ratio of the energies consumed in air and hydrogen respectively is in agreement also with the decrease in the hydrogen equivalent of the celloidin film.

Still making use of our hypothesis, the ratio of the energy consumed in the 9 th and 10 th centimetres of air at reduced pressure, to that consumed in the same centimetres of hydrogen at normal pressure, is expressed by the fraction 312 . The same ratio for the 13 th and 14 th centimetres is $\frac{77}{8 \overline{3}}$. These ratios were obtained by measuring with a planimeter the areas in fig. 2. The former ratio divided by the latter gives $1 \cdot 10$. Since the hydrogen equivalent of the colloidin film is but slightly more than 2 centimetres, the ratio of its values at 9 and $13 \mathrm{~cm}$. respectively from the polonium should be the same as the above ratio. The hydrogen equivalents of the film in the two positions (see fig. 1) are 2.320 and $2.120 \mathrm{~cm}$. respectively ; and the ratio of the former to the latter is $1 \cdot 09$, which differs little from the calculated ratio, $1 \cdot 10$, given above. Hence it is seen that the differences between the curves of fig. 2 are sutficient to account for the change in the hydrogen equivalent of the celloidin film, and consequently for the increase in the air equivalents of the hydrogen sheets when moved away from the source of rays. This agreement between the relative ionizations and the relative losses of energy of the particle in the two gases gives a considerable degree of probability to our hypothesis connecting the relation of the ionization produced to the energy consumed.

The experimental results show that the air-equivalents of the metal sheets decrease with the speed of the alpha-particle;

Phil. Mag. S. 6. Vol. 18. No. 106. Oct. 1909. 2 T 
and hence the ratio of the energy of the alpha-particle consumed by its passage through a sheet of metal, to the energy that would be consumed by, say, 1 centimetre of air at the same point in the path of the particle, decreases as the range of the alpha-particle decreases. The behaviour of the metal sheets relative to the air is entirely analogous to the behaviour. of the air, or celloidin relative to hydrogen. Consequently, if it were possible to measure the ionization produced by the alpha-particle at different points in the path of the rays in the metals, and if the ionization-curves were plotied on the same scale as those shown for air and hydrogen (fig. 2), it is probable that the curves for the metals would all present some such differences from the air curve as those existing between the air and hydrogen curves. Moreover, these differences might be expected to be such as to agree with the different rates at which the air-equivalents of the different metal sheets change. In the upper portion the curve for gold would probably lie within the air curve about the same amount as the air curve does within the hydrogen curve (fig. 2); and in the lower portion the curve for gold would probably lie without the air curve by the same amount as the air curve does without the hydrogen curve. At least some such differences would be in accordance with the square root law, since the square root of the atomic weight of air is a mean proportional between the square roots of the atomic weights of gold and hydrogen. The curves for the other metals would occupy intermediate positions between the curves for gold and air.

We have seen that for different metal sheets of about the same air-equivalents, the rates at which the air-equivalents decrease with the speed of the alpha-particle are proportional to the square roots of the atomic weights of the respective metals. Consequently, the rates of decrease of the ratios of the quantities of energy used up in the sheets to the energy that would be consumed by a centimetre of air at the saine positions in the path of the particle decreases also as the square roots of the atomic weights of the respective metals. On the basis of our hypothesis that for a given speed of the alpha-particle the same amount of energy is required to produce an ion in all substances, and from the results in our experiments, it appears very probable that for the high velocities the alpha-particle loses its energy, in going through a substance, more rapidly the higher the atomic weight of the substance; but as the speed of the alpha-particle becomes less, this changes until for the low velocities the loss of the energy of the particle is more rapid the lower the atomic weight of the substance. 
In conclusion, I wish to express my gratitude to Professor Bumstead, at whose suggestion these experiments were undertaken, for his valuable suggestions and interest in the work; also to Professor Boltwood, who kindly prepared the polonium and secured the radium bromide for me, and gave me many valuable suggestions.

Summary of Results.

1. The air-equivalents of metal foils decrease with the speed of the alpha-particles entering them. The decrease is very small for thin foils of the lighter metals when the speed of the alpha-particle is high ; but when the speed is low for thin sheets, or when the sheets are thicker, the change becomes more marked. For different sheets of the same metal the rates of change are proportional to the airequivalents of the sheets. For sheets of different metals of equal air-equivalents the rates of change are approximately proportional to the square roots of the respective atomic weights.

2. The air-equivalents of hydrogen cells or sheets increase as the speed of the entering particle decreases, while the airequivalents of sheets of paper and celloidin remain constant.

3. The hydrogen-equivalents of sheets of paper, films of celloidin, and air do not remain constant, but decrease as the speed of the alpha-particle decreases. The rate at which the hydrogen-equivalent of a celloidin film decreases with the speed of the entering alpha-particle is numerically equal to the rate at which the air-equivalent of a hydrogen sheet of corresponding thickness increases.

4. The result obtained by Bragg, that the total ionization. produced by the alpha-particle in air is the same as that in hydrogen, is confirmed by a more direct method.

5 . It is very probable that for the high ranges the alphaparticle loses its energy, in passing through substances, more rapidly the higher the atomic weight of the substance; but that this difference decreases slowly, until in the low ranges the loss of energy is the more rapid the lower the atomic weight of the substance.

6. A comparison of the Bragg curves for air and hydrogen indicates that the large ionization at low ranges (knee of the curve) is due at least in part to the fact that the particle loses its energy more rapidly in this part of the range, and not wholly to the higher ionizing efficiency of particles of low speed.

Sloane Laboratory,

Yale University,

New Haven, Conn., U.S.A. 